for patients with AUR and to devote further resources into preventing the condition.

Original article Thomas K et al. (2005) Acute urinary retention: what is the impact on patients' quality of life? $B J U$ Int 95: 72-76

\section{Is total androgen blockade useful in metastatic prostate cancer?}

A recent study by Ansari and colleagues has shown that orchiectomy alone was equally as effective as orchiectomy plus flutamide in the treatment of men with metastatic carcinoma of the prostate.

The study included 100 patients with advanced prostate cancer who were randomized in a 1:1 ratio to orchiectomy alone $(O)$ or orchiectomy plus flutamide (OF), and were followed up for a mean of 3.5 years. The maximum percentage decrease in serum PSA levels was similar in both groups (95\% and $97 \%$ for groups $\mathrm{O}$ and $\mathrm{OF}$, respectively), and occurred during the first 3 months after orchiectomy. This decrease in PSA persisted for 3 years in more than $80 \%$ of patients in both groups. There was no significant difference between the $O$ and OF groups in the proportion of patients experiencing complete or partial response, as indicated by serum PSA levels and bone scan findings. The mean time to progression was 27 and 29 months in the $\mathrm{O}$ and OF groups, respectively, and overall survival was similar in the two groups at 3 years ( $45.8 \%$ vs $48.1 \%)$ and 5 years $(20.8 \%$ vs $23.1 \%)$.

In summary, total androgen blockade was not superior to orchiectomy alone in decreasing serum PSA in this study, and overall survival was similar using the two strategies. The authors, therefore, advise against the routine addition of flutamide in patients undergoing orchiectomy.

Original article Ansari MS et al. (2004) Combined androgen blockade in the management of advanced prostate cancer: a sensible or ostensible approach. Int J Urol 11: 1092-1096

\section{Prostate cancer treatment and osteoporosis risk}

Insufficient attention is given to the risk of osteoporosis in men receiving androgen deprivation therapy (ADT), according to a recent study published in Cancer. Although ADT is a known risk factor for osteoporosis, few patients appear to receive the appropriate interventions for the prevention or treatment of the disease.

Tawee Tanvetyanon carried out a retrospective analysis of 184 men with prostate cancer, all of whom had been receiving ADT with goserelin injection for at least a year. During the previous 3 years, only 16 patients $(8.7 \%)$ had undergone DXA scanning to test bone mineral density. A similar number had received calcium or vitamin $D$ supplements in the past year, while $10(5.4 \%)$ patients received oral or intravenous bisphosphonates. Overall, interventions for the prevention or treatment of osteoporosis were provided to only 27 participants $(14.7 \%)$. Although many of the patients had concurrent risk factors for osteoporosis, such as smoking, advanced age or history of fractures, these patients were no more likely to have received interventions than those without additional risk factors. Patients with bone metastases, however, were over five times more likely to have received at least one intervention than those without. The study also revealed that primary care physicians offered more interventions than did cancer specialists or those from other specialties.

In summary, there is a need to improve prevention, diagnosis and treatment of osteoporosis among men undergoing ADT and, importantly, to increase patient and physician awareness of the problem.

Original article Tanvetyanon T (2004) Physician practices of bone density testing and drug prescribing to prevent or treat osteoporosis during androgen deprivation therapy. Cancer 103: 237-241

\section{Bone-anchored sling safely relieves male SUI}

Stress urinary incontinence (SUI) subsequent to radical prostatectomy is generally related to intrinsic sphincter dysfunction. An artificial urinary sphincter (AUS) is the gold standard approach to management of this problem. In light of the successful use of slings to relieve SUI in women, Rajpurkar and colleagues assessed the performance of a bone-anchored perineal version as an alternative to AUS.
GLOSSARY

DXA

Dual-energy X-ray

absorptiometry 